\title{
Definición del Problema de la Calidad de la Atención Médica y su Abordaje Científico: Un Modelo para la Realidad Mexicana
}

\section{A Scientific Approach to the Definition of Quality in Medical Care: A Model for Mexican Reality}

Armando M. Ramírez ${ }^{1}$

Javier E. G. de A. García²

Sandra R. Fraustro ${ }^{3}$

\section{RAMIREZ, A. M.; GARCIA, J. E. G. A. \& FRAUSTRO, S. R. A Scientific Approach to the Definition of Quality in Medical Care: A Model for Mexican Reality. Cad. Saúde Públ., Rio} de Janeiro, 11 (3): 456-462, Jul/Sep, 1995.

This paper analyzes the problem of quality of medical care and its evolution in various health service institutions. It concludes that quality strategies for countries like Mexico should be of the decentralized/participant type, and that all quality programs in medical care should include five fundamental elements: evaluation, monitoring, design, development, and organizational change.

Key words: Medical Care; Quality; Health Services

\section{INTRODUCCION}

Tradicionalmente, en México, las instituciones públicas de atención a la salud, han utilizado mecanismos de control administrativos del ejercicio médico, cuyos efectos han sido contraproducentes sobre la calidad de la atención médica.

La escasa resistencia al avance del burocratismo en las organizaciones de atención a la salud, expresa el debilitamiento de las instituciones médicas que son incapaces de llevar a un primer plano intereses académicos de superación profesional de los médicos en las unidades hospitalarias en que laboran y, de influir y reorientar las decisiones que se tomen en la cúspide de la pirámide

\footnotetext{
${ }^{1}$ Privada Colomos, 1883 Depto. 201-C Fracc. Colomos Providencia, 44620, Guadalajara, Jalisco, Mexico.

${ }^{2}$ Instituto Regional de Salud Publica, Centro Universitario de Ciencias de la Salud, Universidad de Guadalajara. Guadalajara, Jalisco, México. Prof. Investigador, Universidad de Guadulajara.

${ }^{3}$ Subjefatura de Investigación Epidemiológica, Jefatura de Investigación Médica, Instituto Mexicano del Seguro Social, Guadalajara, Jalisco, Mexico.
}

político-administrativa. Ello permite entender porqué el ejercicio de la medicina adquiere cada vez más la fisonomía de un procedimiento administrativo, que poco tiene que ver con los intereses de los usuarios de los servicios, y transforma al médico en un empleado burocratizado que ha perdido la orientación humanista de su quehacer diario y su actitud inquisitiva hacia el conocimiento (Viniegra-Velázquez, 1990).

Sin embargo, actualmente existe un crecimiento vertiginoso del interés por la calidad de la atención médica en México y en muchos otros países, cuyo origen es probablemente una mayor conciencia de que, en el mundo contemporáneo, la calidad se ha convertido en un requisito indispensable de sobrevivencia económica y, para algunos afortunadamente también, de responsabilidad social y de integridad moral (Ruelas \& Zurita, 1993).

Nuestro país, aunque ha iniciado este largo proceso de cambio de valores todavía le falta mucho camino por recorrer, por to que se necesita, ahora más que nunca, desplegar un mayor esfuerzo en aras de alcanzar esa conciencia y una preparación que nos permita remover arraigadas 
estructuras y abrir así una nueva era de desarrollo, investigación y aprendizaje.

A1 igual que otros países latinoamericanos, México aún enfrenta grandes carencias de recursos, por to que se ha soslayado la cuestión de la calidad de los servicios de salud. Sin embargo, ha llegado la hora de empezar a preguntarse no solamente por la cantidad de servicios que se necesitan, sino también y de manera fundamental, por su tipo y calidad.

De hecho, la aplicación de los servicios de salud se han realizado, principalmente, sobre la base de un modelo de atención que restringe sus fundamentos biológicos individuales, que olvida la promoción de la salud, para centrarse en la reparación de órganos enfermos, que fragmentan la totalidad corporal y social en áreas de un enfoque de subespecialidades que gravitan en torno a una tecnología, muchas veces suntuaria, que encuentra en el hospital su espacio cerrado y jerárquico de operación, y que, en suma, se olvida de las necesidades y demandas explícitas de la mayoría de la población. Ahora bien, en un medio como el médico acostumbrado a centrarse excesivamente en aspectos cuantitativos, el asunto de la calidad y la mayor parte de los intentos para evaluarla, se han limitado al recuento de acciones emprendidas sin plantearse el efecto real que dichas acciones tienen sobre la salud, ni la medida en que satisfacen las necesidades de los individuos y de la población (Frenk, 1978).

Así entonces, los objetivos de la presente comunicación son:

a. Hacer una revisión de algunas consideraciones teóricas sobre calidad de la atención médica que privan en nuestro medio.

b. Formular una propuesta de modelo de garantía de calidad para nuestras instituciones de salud.

\section{METODOS}

Para hacer un análisis más detallado del problema de la calidad de la atención médica se requiere acortarlo (definirlo), para to cual se necesita primero, decidir cual de la información que tenemos disponible será útil, describir su contenido y forma y analizar la factibilidad y viabilidad de su abordaje científico.

Enseguida, debemos formular el estado actual del problema, es necesaria esta etapa para fundamentar hacia el exterior nuestra propuesta, pero además facilita el desarrollo metodológico de la investigación al clarificar y sistematizar el problema de estudio.

Algunos elementos puntuales que debemos de tener en cuenta sobre la información básica y el estado del problema son:

1. Marco referencial.

2. Descripción del problema.

3. Análisis de factores sustantivos.

4. Propuesta teórica de como abordar el problema de estudio.

\section{RESULTADOS}

En principio el problema de la atención médica y por ende de su calidad, parte de una relación entre la cantidad y la calidad de los servicios proporcionados. En efecto, teóricamente si la cantidad de servicios proporcionados es insuficiente, los beneficios esperados tienden a disminuir. Por el contrario, una excesiva cantidad de servicios puede conducir a un incremento en los riegos a los que se expone un paciente. Esta relación entre cantidad y calidad también implica necesariamente una relación entre costos y calidad.

Uno de los autores que ha analizado esta relación es Donabedian (1980) quien señala que cuando la atención es excesiva y dañina, es más costosa y de menor calidad; cuando la atención es excesiva pero no produce daños es también más costosa pero ese costo no corresponde a los beneficios esperados, y ello significa desperdicio; existe, pues, una relación beneficios $\rightarrow$ riesgos $\rightarrow$ costos, en la que el hipotético valor neto de la calidad, podría calcularse al restar a los beneficios, la suma de los riesgos más los costos (Modelo Unificado de la Calidad). Esto nos lleva a concluir que la eficiencia y la calidad son dos componentes indisolubles de la atención a la salud. 
Estas últimas consideraciones son particularmente relevantes a los contextos de los países en desarrollo como México en los cuales, con frecuencia, se argumenta la imposibilidad de otorgar servicios de calidad por la carencia de recursos. En efecto, esta carencia puede afectar la calidad, pero no puede atribuírsele la totalidad del deterioro de la calidad, de ahí que uno de los grandes retos de la evaluación de la calidad derivan de la dificultad de atribuir los resultados realmente a la atención proporcionada, esto significa que no todos los resultados pueden ser fácil ni exclusivamente atribuibles a los procesos, y no todos los procesos dependen directa y unívocamente de la estructura (Donabedian, 1988).

A1 tiempo que se transita de la preocupación por la cantidad hacia la preocupación por la calidad, en los últimos 10 años se ha ido transitando también de los esfuerzos por evaluar la calidad a aquellos por garantizar niveles adecuados de esta a los usuarios de los servicios (Ruelas-Barajas, 1992).

Con base en la evaluación de la calidad surgió la necesidad de la verificación, es decir, la necesidad de constatar el cumplimiento de estándares que aseguraran niveles adecuados de calidad. No obstante, paulatinamente se hizo cada vez más evidente que ni la evaluación per se, ni la acreditación como elemento central para asegurar la calidad, conducen necesaria y automáticamente hacia la corrección de problemas, que disminuían los beneficios esperados o incrementaban un paso más hacia adelante, sobre todo porque estos elementos se transformaron en fines por sí mismos, fue así que la transición de la evaluación a la garantía se hizo necesaria (Ruejas-Barajas, 1990).

Ya desde 1986, se señalaba que garantía de la calidad "Son aquellas actividades que periódica o contínuamente se dirigen a revisar las condiciones bajo las cuales se proporciona ateneión médiea, esta es monitorizada y los resultados son seguidos de manera que las deficiencias sean descubiertas y se hagan las correcciones necesarias" (Donabedian, 1986: 43). Así para Donabedian, existen dos elementos centrales para garantizar la calidad:

Monitoría y un adecuado diseño del sistema de salud (Donabedian, 1986).

En México, como resultado de la primera experiencia de garantía de calidad, Ruelas-Barajas \& Reyes-Zapata (1990: 178) con el objeto de operacionalizar una definición proponen que la "Garantía de la calidad es un conjunto de acciones sistematizadas y continuas tendientes a prevenir y/o resolver oportunamente problemas o situaciones que impidan el mayor beneficio o que incrementen los riesgos a los pacientes, a través de cinco elementos fundamentales: Evaluación, monitorea, diseño, desarrollo y cambio organizacional" (Figura 1).

En efecto, la evaluación es el pilar fundamental para garantizar la calidad de la atención. No es posible mejorar algo cuyo comportamiento se desconoce. La monitoréa (entendida como medición sistematizada del comportamiento de una o varias variables durante periodos establecidos y con frecuencias determinadas), es también un componente indispensable de la garantía, ya que se trata de establecer continuidad de manera cíclica en la observación del proceso de la atención y sus resultados con el objeto de dar la oportunidad requerida para la identificación y corrección de las situaciones que pudieran afectar el proceso.

Ahora bien, el diseño organizacional puede ser definido como la combinación de los elementos de la estructura organizacional que tiende a asegurar un adecuado desempeño de la propia organización. En el diseño organizacional están comprendidos: La asignación y distribución de la autoridad, definición de responsabilidades, mecanismos de comunicación y coordinación, grados de formalización y estandarización de los procesos, mecanismos de control y tipos de incentivos. Como puede apreciarse, una gran cantidad de problemas que impactan directamente a la calidad de la atención se derivan de diseños organizacionales defectuosos. 
El desarrollo organizacional es una disciplina que se define como el establecimiento de estrategias educativas quepermiten a los individuos hacer conciencia explícita de los objetivos de la organización, de la importancia de su propio trabajo, y del de los demás para la consecución de esos objetivos, de la manera en que pueden mejorar su desempeño y el de los demás y del desarrollo del liderazgo en la organización.

FIGURA 1. Elementos de un Programa de Garantia de Calidad

\section{ESTRATEGIA DE GARANTÍA DE CALIDAD}

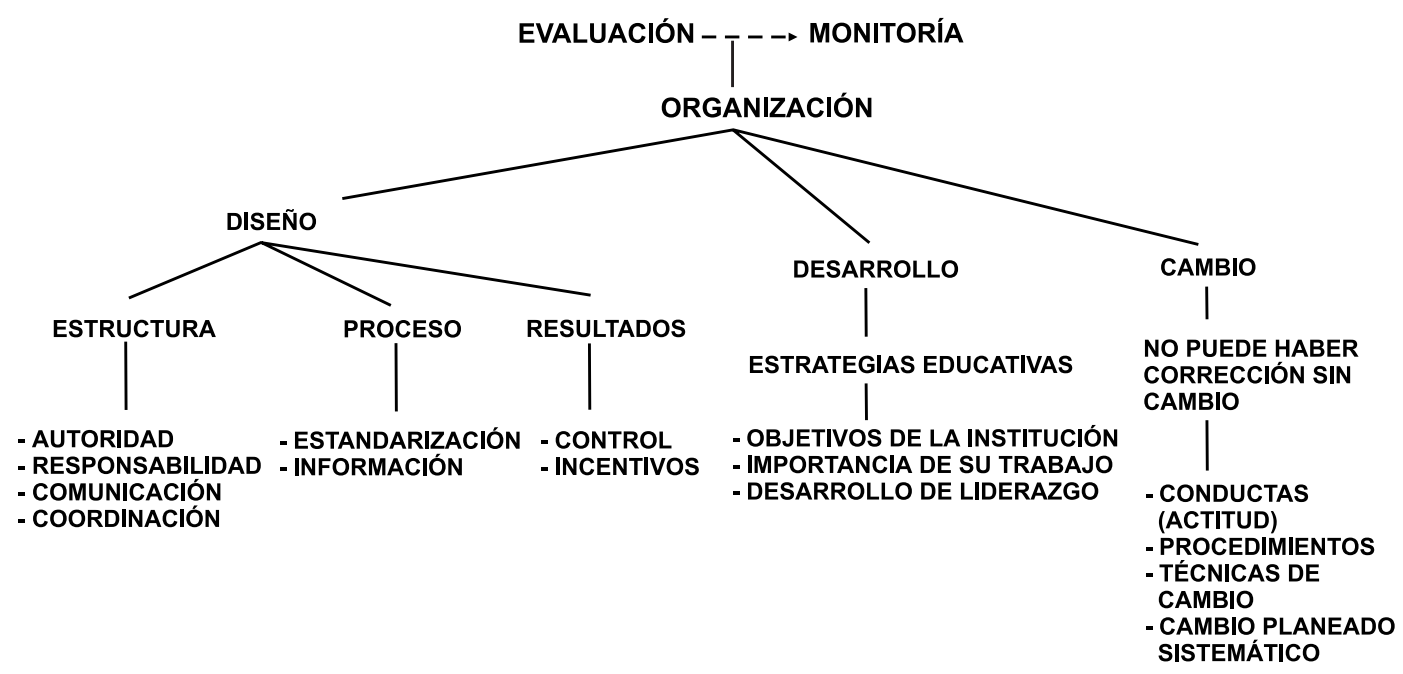

No puede haber corrección sin cambio. El cambio en este caso, puede ser de conductas o procedimientos que, a su vez, pueden implicar cambios de actitud, debemos entender to que significan diferentes tipos de cambio en las organizaciones y las técnicas que puedan facilitarlo, entre ellas el desarrollo organizacional es fundamental para garantizar la calidad de la atención.

Todo lo anterior se operacionaliza a través de programas de garantía de calidad que nos permitan sistematizar las acciones, identificar problemas oportunamente, analizarlos objetivamente, proponer soluciones factibles y dar seguimiento a las acciones propuestas. Los principales requisitos para ello son: Indicadores y estándares y un sistema de información adecuado, entendido este no sólo como captura y procesamiento de datos y generación de información "Hacia arriba" como frecuentemente sucede, sino como un sistema de retroalimentación, y mecanismos de seguimiento que aseguren que las correcciones se han llevado a cabo (RuelasBarajas, 1992) (Figura 2).

Diferentes tipos de programas de garantía de calidad podrían ser clasificados de acuerdo con la ubicación organizacional de las instancias que generan los estándares que monitorizan y evalúan el desempeño manifestado por dichos estándares y que deciden sobre las medidas correctivas a adoptar, y de acuerdo con el grado de participación de los diferentes individuos 
FIGURA 2. Actividades de un Programa de Garantia de Calidad



involucrados en el proceso de atención. De aquí surgen tres tipos posibles: Programas de garantía de calidad centralizados, descentralizados no participativos y descentralizados participativos (Ruelas-Barajas \& Frenk, 1989).

Por otra parte, se señala que para lograr la participación de los trabajadores en forma efectiva, es importante realizar esfuerzos sostenidos de educación, para crear la sensibilidad necesaria hacia los aspectos de la calidad y adquirir la competencia para manejarla (Donabedian, 1992), esto requiere de una perspectiva epidemiológica y de las correspondientes herramientas descriptivas y de análisis.

Cabe hacer énfasis en reformar el proceso de trabajo y el contexto en que se desarrolla. Estos hechos estructurales, y los incentivos que engloban, deberían facilitar y asimilar una buena actividad más que poner obstáculos para lograrlo. En particular la tentación de culpabilizar y penalizar, tan difundida por las regulaciones gubernamentales, debiera contrastar con nuestras mejores tradiciones profesionales de autoevaluación y aprendizaje continuo.

Más allá de sus orígenes o afiliaciones, todos nuestros modelos de garantía de calidad claman por una investigación científica más rigurosa. Para empezar, nos podrían ayudar a identificar cualquier enfoque dentro de las amplias teorías de conducta y efectividad organizacional. Esto ayudaría a desarrollar un conjunto de modelos más diferenciados, como un primer paso para una exploración empírica de su desempeño. Las pruebas empíricas serán, por supuesto, de dos formas: Una observacional, empleando los métodos epidemiológicos y la otra experimental, usando las posibilidades de los ensayos y trabajos de cámpo. De esta forma, a partir de la construcción de un modelo y de su evaluación empírica, estableceremos un objetivo concreto: El estudio comparativo de sistemas de garantía de calidad.

\section{DISCUSION}

Una vez analizado todo este bagaje de conocimientos teóricos, consideramos que el programa de garantía de calidad que debemos desarrollar en los establecimientos médicos prestadores de servicios públicos de salud en países como el nuestro, deben ser, de tipo descentralizado-participativo, cuyas características esenciales sean: El establecimiento de una responsabilidad 
compartida entre todos los miembros de la organización para mejorar contínuamente la calidad; considerar como usuario no sólo al consumidor, sino a los propios miembros de la organización; analizar el sistema en su conjunto y no sólo a los individuos como posibles causantes de errores en los procesos y defectos en los resultados; un esfuerzo contínuo para mejorar, en lugar de una adherencia pasiva a estándares pre-establecidos rígidos, utilizar las técnicas estadísticas para monitorizar los procesos y los resultados a fin de producir acciones informadas que corrijan desviaciones, pasar de un predominio de las jerarquías a un enfoque eminentemente participativo; de un liderazgo exclusivamente gerencial a un liderazgo compartido con los profesionales de la salud que proporcionan los servicios directos a pacientes y familiares; de una responsabilidad individual por la calidad a una responsabilidad compartida por todos los miembros de la organización; de esquemas rígidos de organización y gestión a enfoques flexibles; de evaluaciones retrospectivas a evaluaciones concurrentes (Figure 3).

FIGURA 3. Características de un Programa de Garantia de Calidad

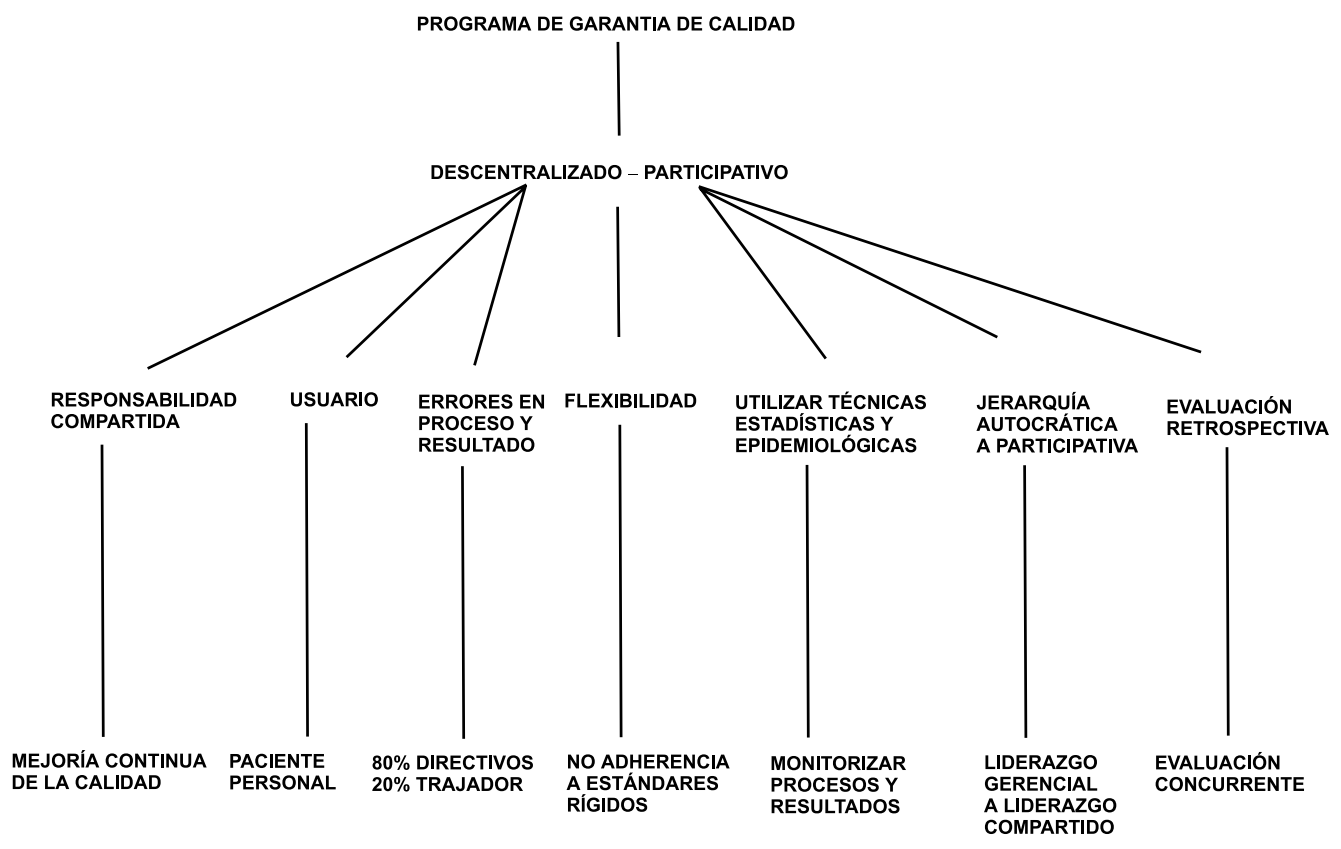

Sabemos que esto implica un proceso tal vez largo, de capacitación y motivación sobre técnicas y estrategias de garantía de calidad que conduzan a resultados observables sobre los cuales la monitoréa y la evaluación surjan como consecuencia y no viceversa.

Estamos conscientes que es fundamental capacitar antes de exigir. Todo esto requiere además incorporar al proceso, a la investigación cualitativa y cuantitativa que permita, no solo monitorizar la calidad, sino la efectividad y la eficiencia de los esfuerzos de garantía de calidad y que valide científicamente los resultados.

\section{RESUMEN}

RAMIREZ, A. M.; GARCIA, J. E. G. A. \& FRAUSTRO, S. R. Definición del Problems de la Calidad de la Atención Médica y su Abordaje Científico: Un Modelo pare la Realidad Mexicana. Cad. Saúde Públ., Rio de Janeiro, 11 (3): 456-462, jul/set, 1995. 
El presente estudio tiene como objetivo analizar el problema de la calidad de la atención médica en México, así como la evolución de la misma en las diferentes instituciones prestadoras de servicios de salud, y concluye que las estrategias de garantía de calidad de tipo descentralizado-participativo son la mejor opción para abordar el problema de la calidad en países como el nuestro, y que cualquier programa de calidad debe incluir cinco elementos fundamentales: La evaluación. la monitoréa, el diseño, el desarrollo y el cambio organizacional.

Palabras Clave: Atención Médica: Calidad; Servicios de Salud: Salud Pública

\section{REFERENCIAS BIBLIOGRAFICAS}

DONABEDIAN, A.. 1980. The definition of quality and approaches to its assessment. Ann. Arbor, Mich.: Health Administation Press, VOL 1: 38-40.

1986. Quality assurance in our health care system. Quality Assurance Utilization, 1: 37-68.

1988. Veinte años de investigación en torno a la calidad de atención médica, 1964-1984. Salud Pública de México, 30: 202-215.
1992. Continuidad y cambio en la bú queda de la calidad. Salud Pública de México (Supt. 35): 85-104.

FRENK, J., 1978. Comentario al artículo de Avedis Donabedian "The Quality of Medical Care". Science, 200: 856-864.

RUELAS, B. E. \& ZURITA, G. B., 1993. Nuevos horizontes de la calidad de la atención a la salud. Salud Pública de México, 35: 235-237.

RUELAS-BARAJAS, E., 1990. De la cantidad a la calidad y de la evaluación a la garantía. Salud Príblica de México, 32: 108-109.

1992. Hacia una estrategia de garantía de calidad. de los conceptos a las acciones. Salud Pública de México, 34: 29-45.

RUELAS-BARAJAS, E. \& FRENK J., 1989. Frame work for the analysis of quality assurance in transition: The case in Mexico. Australian Clinical Review, 9: 09-16.

RUELAS-BARAJAS, E. \& REYES-ZAPATA, H., 1990. Círculos de calidad como estrategia de un programa de garantía de calidad de la atención médica en Instituto Nacional de Perinatología. Salud Pública de México, 32: 207-220.

VINIEGRÁ-VELAZQUEZ, L., 1990. El control de calidad del ejercicio de la medicina: un marco teórico. Gaceta Médica de México, 126: 283-289. 